\title{
Potential of Carbon Nanotubes in Enhance of Photocatalyst Activity
}

\author{
Atheel Alwash ${ }^{1}$, Hadeel Adil' ${ }^{1}$, Zainab Hussain ${ }^{2}$ and Emad Yousif ${ }^{1 *}$ \\ ${ }^{1}$ Department of Chemistry, College of Science, Al-Nahrain University, Baghdad, Iraq \\ ${ }^{2}$ Department of Chemistry, College of Science, Misan University, Misan, Iraq
}

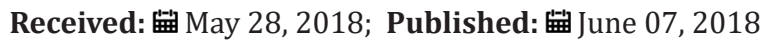

*Corresponding author: Emad Yousif, Department of Chemistry, Al-Nahrain University, College of Science, Baghdad 10072, Iraq

\begin{abstract}
Carbon nanotubes (CNTs) have generated huge activity in most areas of science and engineering due to their remarkable physical and chemical properties. No previous materials have displayed the combination of superlative mechanical, thermal and electronic properties attributed to them. These properties make nanotubes ideal, not only for a wide range of applications but as a test bed for fundamental science. This review highlighted some of the properties of these nanotubes and also their role as supports materials in enhance of photocatalytic activity of nanocrystals for removal of contaminants.
\end{abstract}

Keywords: Carbon Nanotubes; Photocatalytic Activity; Nanocrystals; Hybridization; Honeycomb Atomic Arrangement; Tubular Carbon Structures; Multi-Walled Carbon Nanotubes; Single-Walled Carbon Nanotubes; Atomic Vacancies; Ballistic Conduction

\section{Introduction}

Elemental carbon in the $\mathrm{sp}^{2}$ hybridization can form a variety of amazing structures. Apart from the well-known graphite, carbon can build closed and open cages with honeycomb atomic arrangement. First such structure to be discovered was the C60 molecule by Kroto [1]. Although various carbon cages were studied, it was only in 1991, when Iijima [2] observed for the first time tubular carbon structures. The nanotubes consisted of up to several tens of graphitic shells (so-called multi-walled carbon nanotubes (MWNTs)) with adjacent shell separation of $\sim 0.34 \mathrm{~nm}$, diameters of $\sim 1 \mathrm{~nm}$ and large length/diameter ratio. Two years later, Iijima and Ichihashi [3] and Bethune et al. [4] synthesized single-walled carbon nanotubes (SWNTs). Nowadays, MWNTs and SWNTs are produced mainly by three techniques: arc-discharge, laser-ablation, and catalytic growth [5] Figure 1 shows these two categories of CNTs) (Figure 1).
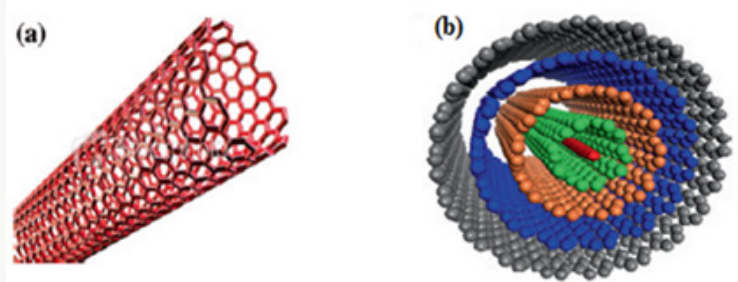

Figure 1: Basic structures of a) single-walled and b) multi-walled CNTs.
CNTs have unique properties that make them potentially useful in a wide variety of applications including applications in nano-electronics, optics, and materials applications [6]. Since the beginning of the $21^{\text {st }}$ century, they have been introduced in pharmacy and medicine for drug delivery system in therapeutics Because of their high surface area, excellent chemical stability, and rich electronic polyaromatic structure [7]. Also, because of unique electronic and adsorption properties of CNTs, many scientifics reported that CNTs could enhance the photocatalytic activity of semiconductors in the treatment of organic contaminants [8].

\section{Properties of Carbon Nanotubes}

\section{Strength}

CNTs have a higher tensile strength than steel. Their strength comes from the $\mathrm{sp}^{2}$ bonds between the individual carbon atoms. This bond is even stronger than the $\mathrm{sp}^{3}$ bond found in diamond. Under high pressure, individual nanotubes can bond together, trading some $\mathrm{sp}^{2}$ bonds for $\mathrm{sp}^{3}$ bonds. This gives the possibility of producing long nanotube wires. CNTs are not only strong, they are also elastic. You can press on the tip of a nanotube and cause it to bend without damaging to the nanotube and the nanotube will return to its original shape when the force is removed. A nanotube's elasticity does have a limit, and under very strong forces, it is possible to permanently deform to shape of a nanotube. 
A nanotube's strength can be weakened by defects in the structure of the nanotube. Defects occur from atomic vacancies or a rearrangement of the carbon bonds. Defects in the structure can cause a small segment of the nanotube to become weaker, which in turn causes the tensile strength of the entire nanotube to weaken. The tensile strength of a nanotube depends on the strength of the weakest segment in the tube similar to the way the strength of a chain depends on the weakest link in the chain [9].

\section{Electrical Conductivity}

A metallic CNT can be considered as highly conductive material. Chirality, the degree of twist of graphene sheet, determines the conductivity of CNT interconnects. Depending on the chiral indices, CNTs exhibit both metallic and semiconducting properties. The electrical conductivity of MWNTs is quite complex as their interwall interactions non-uniformly distribute the current over individual tubes. However, a uniform distribution of current is observed across different parts of metallic SWNT. Electrodes are placed to measure the conductivity and resistivity of different parts of SWNT rope. The measured resistivity of the SWNT ropes is in the order of $10-4 \Omega \mathrm{cm}$ at $27^{\circ} \mathrm{C}$, indicating SWNT ropes to be the most conductive carbon fibers. It has been reported that an individual SWNT may contain defects that allows the SWNT to behave as a transistor [10].

\section{Thermal Properties}

All nanotubes are expected to be very good thermal conductors along the tube, exhibiting a property known as "ballistic conduction," but good insulators laterally to the tube axis. It is predicted that carbon nanotubes will be able to transmit up to $6000 \mathrm{~W} \cdot \mathrm{m}-1$.K-1 at room temperature; compare this to copper, a metal well-known for its good thermal conductivity which transmits 385 W.m-1 .K-1. The temperature stability of carbon nanotubes is estimated to be up to $2800{ }^{\circ} \mathrm{C}$ in vacuum and about $750{ }^{\circ} \mathrm{C}$ in air. Thermal expansion of CNTs will be largely isotropic, which is different than conventional graphite fibers, which are strongly anisotropic. This may be beneficial for carbon-carbon composites. It is expected that low-defect CNTs will have very low coefficients of thermal expansion $[11,12]$.

\section{Carbon Nanotubes in Photocatalysis}

Photocatalysis has been a hot topic in the degradation of organic pollutants for several decades [13]. Traditional photocatalysts include $\mathrm{TiO}_{2}, \mathrm{CdS}, \mathrm{Fe}_{2} \mathrm{O}_{3}, \mathrm{ZnO}$. These semiconductors suffer from some disadvantages: $\mathrm{TiO}_{2}$ has a large band gap and can only be excited by ultraviolet light, thus could not effectively harvest the spectrum of sunlight; $\mathrm{CdS}$ and $\mathrm{ZnO}$ hold the drawback of photocorrosion and cause the decrease in photoactivity and stability; in addition, all the semiconductors have the bottleneck of low quantum efficiency due to the rapid recombination of photogenerated electrons and holes, i.e., most charges quickly recombine without participating in photocatalytic reactions. Owing to their excellent mechanical, electrical and optical properties CNTs can serve as an ideal building block in hybrid catalysts and improve the performance of photocatalysts. CNT can be either semiconducting or metallic depending on their diameter and chirality. CNTs have a large electron-storage capacity and it was estimated that every 32 carbon atoms in SWCNTs can store an electron [14].

When in contact with $\mathrm{TiO}_{2}$ nanoparticles, CNTs prompt electron transfer from the conducting band of $\mathrm{TiO}_{2}$ to the CNT surface due to their lower Fermi level. Thus, CNTs accept and store photogenerated electrons and inhibit the recombination of electrons and holes. Those electrons can be transferred to another electron acceptor, such as molecular oxygen, forming reactive oxygen species $\mathrm{CO}_{2}$ , $\mathrm{H}_{2} \mathrm{O}_{2}$ and $\mathrm{OH}$ ) which degrade and further mineralize organic pollutants. Recent research highlighted the attraction of CNTs to $\mathrm{TiO}_{2}$ nanoparticles. $\mathrm{CNT} / \mathrm{TiO}_{2}$ composites showed enhanced photocatalytic oxidation activity to phenol due to reduced charge recombination as evidenced by the diminished photoluminescence intensity, and SWCNT enhanced the photocatalytic activity of $\mathrm{TiO}_{2}$ better than MWCNT because there are more individual contact between the SWCNT and the $\mathrm{TiO}_{2}$ nanoparticle surface [15].
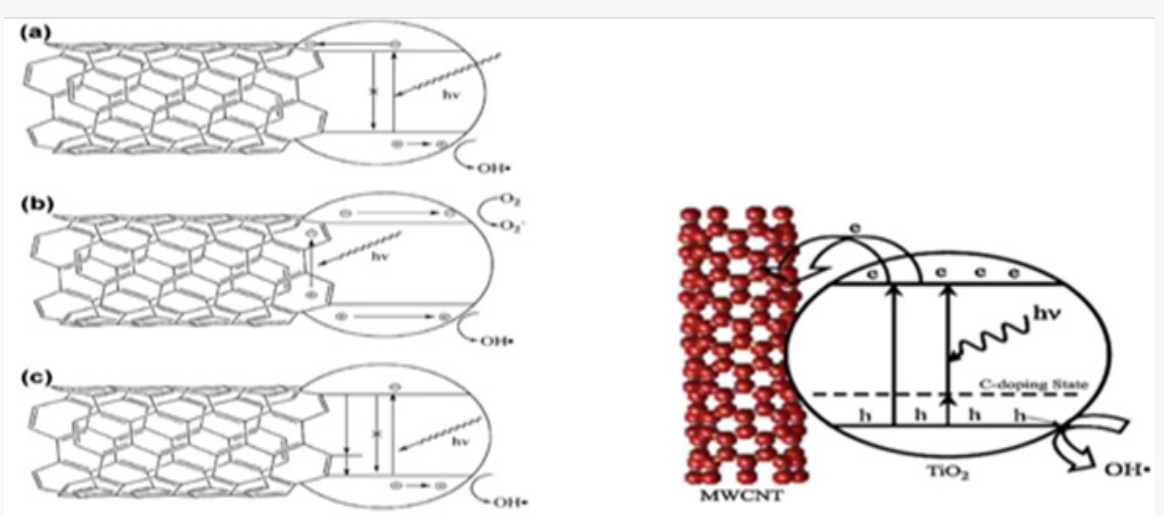

Figure 2: Proposed mechanisms of synergistic enhancement in $\mathrm{TiO}_{2}-\mathrm{CNT}$ composites: (a) CNTs inhibit charge recombination by acting as sinks for photogenerated electrons in $\mathrm{TiO}_{2}$. (b) Photosensitizing mechanism based on electron-hole pair generation in the CNT. Depending on the relevant positions of the bands, the electron or hole may be injected into the $\mathrm{TiO}_{2}$, generating reactive oxygen species. (c) CNTs act as dopants through the Ti-O-C bonds. 
In addition to the inhabitation of charge recombination, the introduction of CNTs increases the amount of hydroxyl groups on the catalyst surface, which can be oxidized by $\mathrm{h}+$ and generate hydroxyl radicals [16]. Aqueous pollutants including dyes [17] benzene derivatives [18] and carbamazepine [19] were efficiently photodegraded by $\mathrm{CNT}^{-T i O}{ }_{2}$ composites. More practically, CNT/ $\mathrm{TiO}_{2}$ composite has found application in the degradation of nitro phenols from real wastewater under sunlight and the composite held repetitive photocatalytic activity [20]. The addition of CNT to $\mathrm{TiO}_{2}$ may change the absorption spectrum of the catalyst. Annealing of CNTs coated with thin and uniform $\mathrm{TiO}_{2}$ results in carbon diffusion into oxide phase via oxygen lattice substitution. Carbon doping produced a mid band-gap state close to the $\mathrm{TiO}_{2}$ valence band and extended light absorption to the visible region (Figure 2) [21]. Considering their semiconductor property, CNTs may also act as photo-sensitizers and inject the photo-excited electrons to the conducting band of $\mathrm{TiO}_{2}[22]$.

Sadeghian S [23] was synthesize titanium dioxide thin films and $\mathrm{CNT}^{-\mathrm{TiO}_{2}}$ nanocomposite using sol-gel method, and then characterized the samples by XRD diffraction pattern, dispersive micro-raman backscattering spectroscopy and UV-Vis absorption and transmission spectroscopies. Results show the presence of only titanium dioxide anatase phase. The preferred peak of crystal growth of $\mathrm{TiO}_{2}$ anatase is (101). X-ray diffraction pattern of CNT$\mathrm{TiO}_{2}$ nanocomposite indicates the overlapping of (101) planes of titanium dioxide and (002) planes of carbon nanotube. By comparing the grain sizes of thin film and nanocomposite, we can see the decreasing of the particles, due to the presence of CNTs. By increasing the thickness of thin films, the grain sizes are also increased. By increasing the thickness of titanium dioxide thin films, the transmission peak shifts to the higher wavelengths. This may be due to the change in the optical energy bandgap in thicker layers. Relatively high transmission through the film indicates low roughness and high uniformity of the film's surface.

Producing the nanocomposite, improves the crystal structure of $\mathrm{TiO}_{2}$ and we observe that the Eg peak related to anatase dioxide titanium, is sharper. By comparing UV-Vis absorption spectra of $\mathrm{TiO}_{2}$ and $\mathrm{CNT} / \mathrm{TiO}_{2}$ nanocomposite, we can see a blue shift in absorption band of the nanocomposite. This may have occurred due to the addition of CNTs to $\mathrm{TiO}_{2}$ and decreasing in particle sizes. By addition of CNTs, we observe an increasing in absorption in visible region of light and increasing of surface charge and enhancement of photocatalytic activity of $\mathrm{CNT} / \mathrm{TiO}_{2}$ (Figures 3-6). Appropriate synthetic approaches are needed for the improvement of the CNT/ $\mathrm{TiO}_{2}$ interface. Eder [24] used benzyl alcohol (BA) as surfactant in the preparation of $\mathrm{CNT} / \mathrm{TiO}_{2}$ composite. The addition of $\mathrm{BA}$ could improve the dispersion state of $\mathrm{TiO}_{2}$ on CNTs through $\pi-\pi$ interaction between BA and CNT and coordination between BA and titanium. Increasing the amount of BA also helped to reduce the size of the deposited $\mathrm{TiO}_{2}$ particles upon crystallization. Some studies also emphasized on the contact resistance of $\mathrm{CNT} / \mathrm{TiO}_{2}$ in terms of electron transportation. SWCNT can enhance the photocatalytic activity of $\mathrm{TiO}_{2}$ more than MWCNT due to more individual contact, but the resistance of SWCNT is high, so there is a need to reduce the interface charge transfer resistance of SWCNT/ $/ \mathrm{TiO}_{2}$.

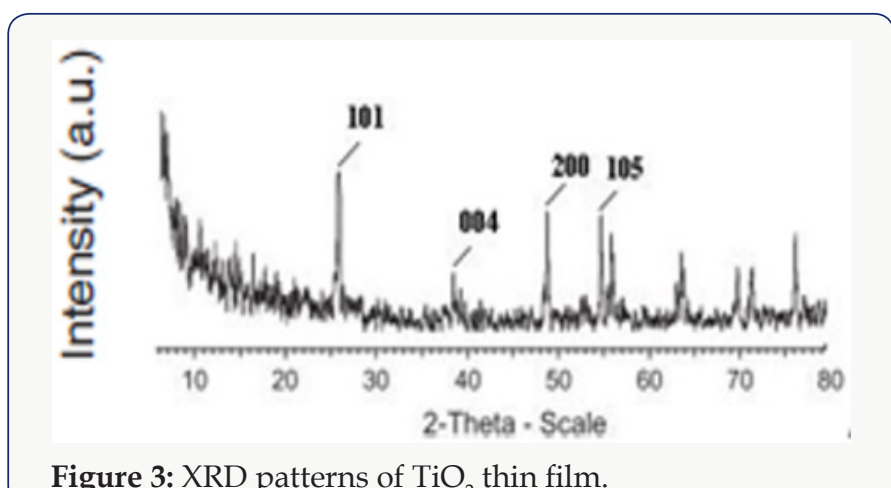

Figure 3: XRD patterns of $\mathrm{TiO}_{2}$ thin film.
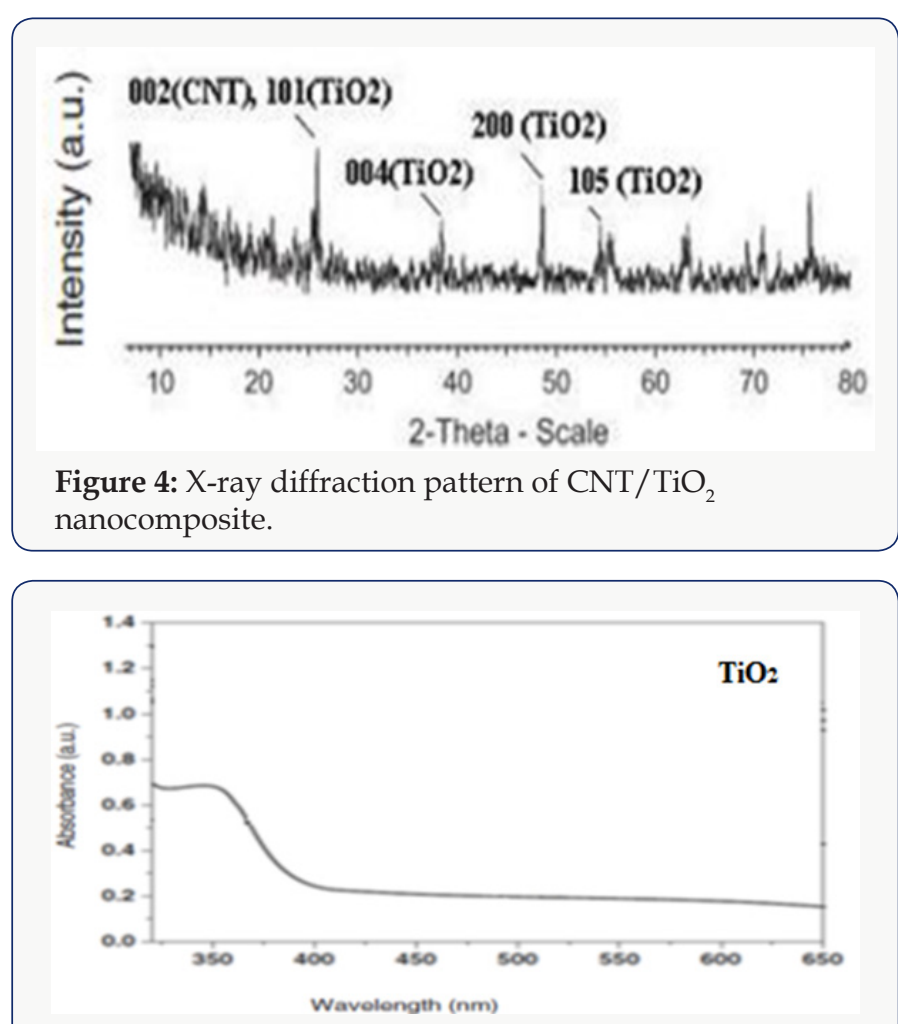

Figure 5: UV absorption spectrum of $\mathrm{TiO}_{2}$ thin film.

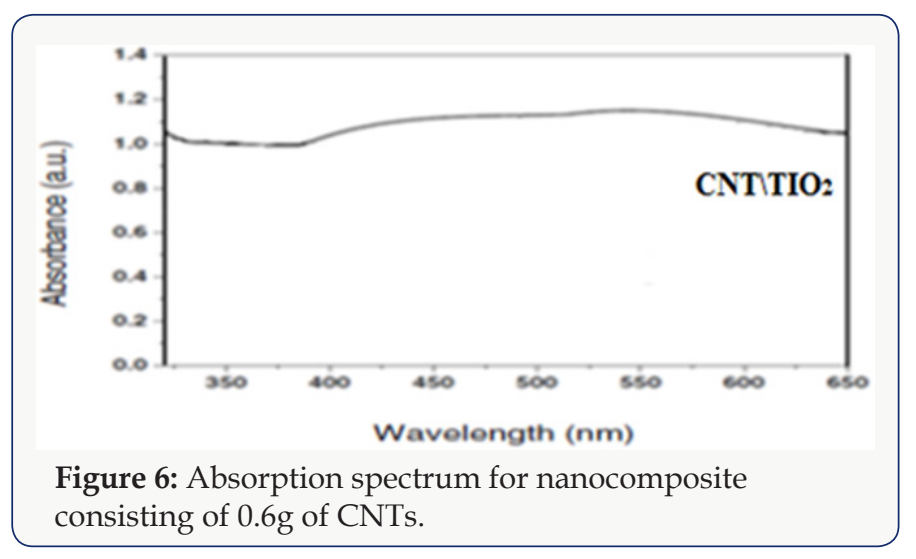

CdS is a visible light-responsive photocatalyst, but it suffers from photocorrosion. Anchoring CdS onto CNTs inhibited the 
photocorrosion phenomenon, and the author attributed it to the enhanced adsorption capacity toward reducing agents in the solution, which can capture holes and stabilize CdS [25]. Applications of CNTs in photocatalysis also include using CNTs as pillars of reduced graphene oxide platelets for rhodamine $\mathrm{B}(\mathrm{RhB})$ degradation [26], and the preparation of Au NP@POM-CNT tricomponent hybrid photocatalyst [27]. Very interestingly, although Au nanoparticles are visible light photo-sensitizers, they don't hold photocatalytic activity, due to the fast rate of charge recombination.
The excellent electron-conducting ability of CNTs made the Au NP@POM-CNT hybrid an effective visible light photocatalyst. It is worth noting that CNTs can absorb the incident light, thus excess CNTs may have an adverse impact on the activity of the composite photocatalyst. Therefore, it is critical to control the dosage of CNTs to photocatalysts [28]. Moreover, the interfacial contact between CNTs and metallic semiconductors dictate the performance of the hybrid photocatalyst.

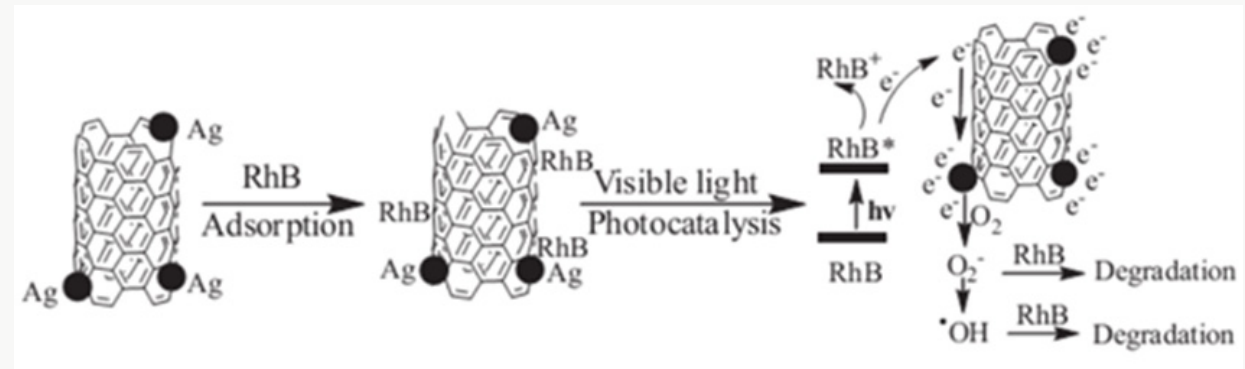

Ag/MWNTs

$\mathrm{RhB} / \mathrm{Ag} / \mathrm{MWNTs}$

$\mathrm{RhB}$ Degradation

Figure 7: Photocatalytic mechanism of RhB degradation on Ag/CNT.

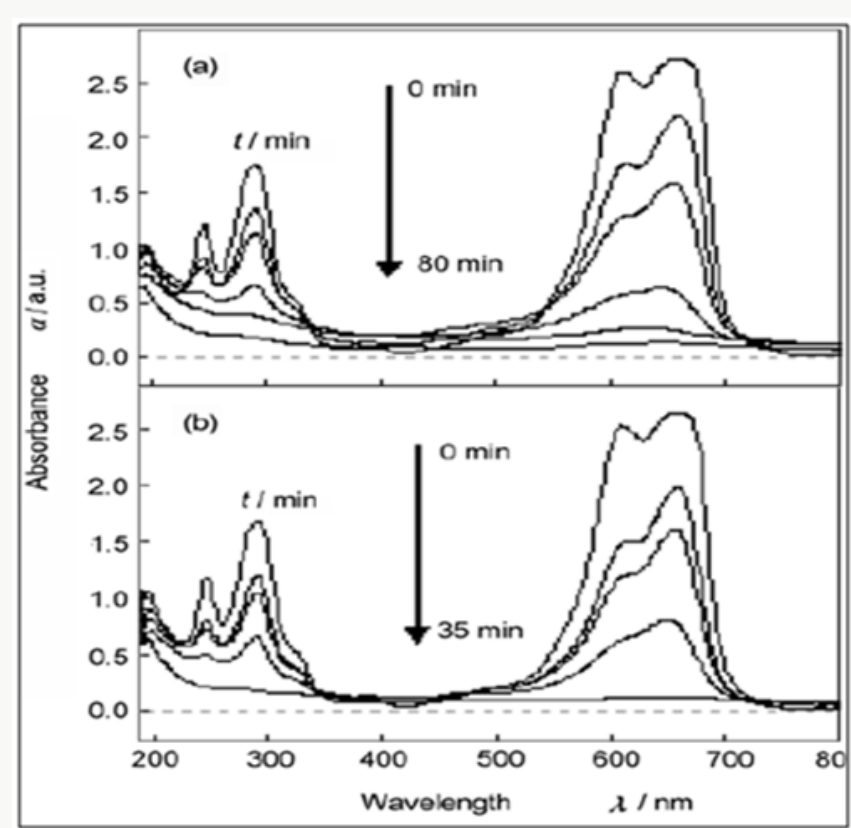

Figure 8: (a) Absorption spectrum of a solution of methylene blue $(\mathrm{MB})$ in the presence of pure ZnS NCs (180mg) under exposure to UV light; (b) Absorption spectrum of a solution of MB in the presence of ZnS/CNTs $\left(\mathrm{Zn}^{2+}-\mathrm{S}^{2-}\right.$, refluxing) $(180 \mathrm{mg})$ under exposure to UV light.

CNTs can also directly act a photocatalyst when decorated with metal. Yan et al. [29] reported that the loading of Ag to CNTs obviously enhanced the photocatalytic activity of CNTs. The Ag/CNT composite exhibited photocatalytic degradation activity toward $\mathrm{RhB}$ and the mechanisms are shown in Figure 7 [29]. First the RhB molecule was adsorbed to CNTs, and then RhB was excited upon visible light illumination. The photo-generated electrons could be transferred along CNT surface and trapped by Ag particles. These trapped electrons reduced the adsorbed oxygen to superoxide anion radicals, leading to the further degradation of RhB (Figure 7). Shou-ai F [30] was synthesize ZnS\CNTs nanocomposite. They find that during the preparation a post-refluxing treatment and a suitable introduction order of reactants are important for the interaction between $\mathrm{ZnS}$ and CNTs. Furthermore, they demonstrate the promoting effect of CNTs on the photocatalytic activity of ZnS NCs by using methylene blue (MB) degradation as the probe reaction. For this promotion, close contact between $\mathrm{ZnS}$ and carbon nanotubes can improve the interfacial electron transfer and restrain the e-/h+ pair recombination of $\mathrm{ZnS}$, by which photocatalytic activity is enhanced (Figures $8 \& 9$ ).

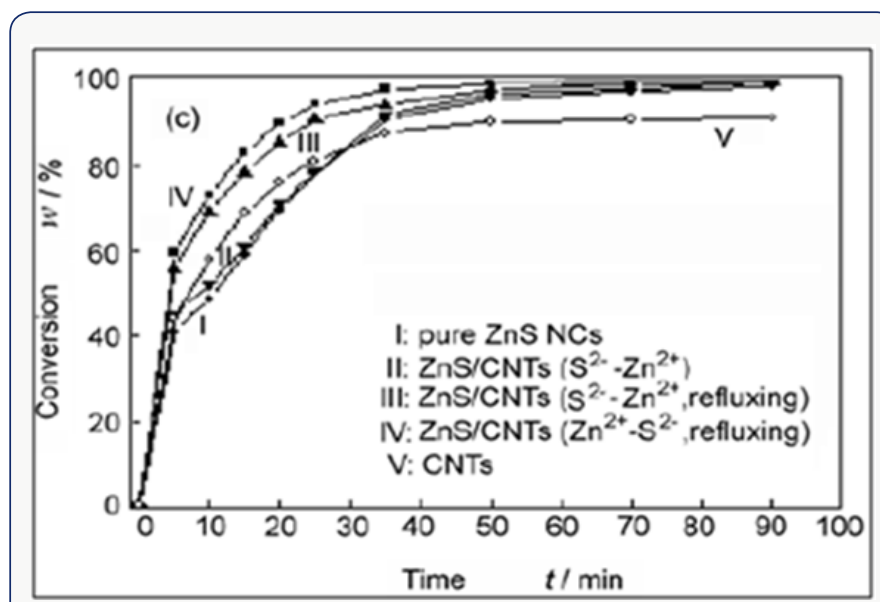

Figure 9: Conversion of $\mathrm{MB}$ as a function of degradation time under exposure to UV light with different conditions: (I) with pure ZnS NCs (180mg); (II) with ZnS/CNTs (S2$\left.-\mathrm{Zn}^{2+}\right)$ (180mg); (III) with ZnS/CNTs $\left(\mathrm{S}^{2-}-\mathrm{Zn}^{2+}\right.$, refluxing) (180mg); (IV) with ZnS/CNTs $\left(\mathrm{Zn}^{2+}-\mathrm{S}^{2-}\right.$, refluxing) (180mg) and (V) with pure CNTs (180mg). 
From Figure (9), the data in curves I and II, it is clear that the photocatalytic activity of $\mathrm{ZnS} / \mathrm{CNTs}\left(\mathrm{S}^{2-}-\mathrm{Zn}^{2+}\right)$ is almost the same as pure $\mathrm{ZnS}$ NCs. As a comparison, ZnS/CNTs $\left(\mathrm{S}^{2-}-\mathrm{Zn}^{2+}\right.$, refluxing) shows higher photocatalytic activity, which indicates that the interaction between CNTs and ZnS NCs is important to enhance the photocatalytic activity of ZnS NCs. This conclusion is further confirmed by the result that $\mathrm{ZnS} / \mathrm{CNTs}\left(\mathrm{Zn}^{2+}-\mathrm{S}^{2-}\right.$, refluxing), where the interaction between CNTs and ZnS NCs is very strong, has the highest photocatalytic activity among these four photocatalysts. The corresponding mechanism can be described as follows: under UV light irradiation, the valence band electrons of $\mathrm{ZnS}$ are excited and move toward its conduction bands, giving rise to the formation of electron and hole pairs. Due to the strong interfacial connection between ZnS NCs and CNTs, the excited e- of the conduction band of ZnS NCs can migrate to CNTs, which are relatively good electron acceptors [31-32], so the recombination of the e-/h+ pairs is retarded, which results in the promotion of photocatalytic activity in ZnS NCs. Another possible improvement of the dispersion of ZnS NCs and thus the enhancement of the photo absorption efficiency of ZnS NCs. This clearly suggests the relationship between the morphology and performance of the different composite samples used. To understand the possible contribution of MB adsorption on CNT, associated with its tubular structure, they have performed a blank experiment on pure CNTs. Although the employed amount of CNTs (180mg), which is the same as that of other photocatalysts in their experiments, is nine times that contained in the ZnS/CNTs composite, and the reduced MB in the solution can reach only $90 \%$ of the initial concentration, suggesting that the fast completed elimination of $\mathrm{ZnS} / \mathrm{CNTs}\left(\mathrm{Zn}^{2+}-\mathrm{S}^{2-}\right.$, refluxing) is mainly associated with other roles rather than CNT adsorption.

\section{Conclusion}

This review was to clarify the role of CNTs in promoting the photocatalytic activity of nanocrystal photocatalyst (oxides or sulfides of semiconductors) in the elimination of organic pollutants. It has been concluded from previous experiments that the strong interfacial connection between photocatalyst and CNTs will promote interfacial electron transfer from the attached nanocrystal photocatalyst to the nanotubes and then retards the recombination of the $\mathrm{e}-\mathrm{h}^{+}$pairs of nanocrystal photocatalyst. Additionally, CNTs as catalyst support can prevent nanocrystal photocatalyst from aggregating, which may be another factor for enhancing the photocatalytic efficiency of this photocatalyst.

\section{References}

1. HW Kroto, JR Heath, SC O'Brien, RF Curl, RE Smalley (1985) C60: buckminsterfullerene. Nature 318: 162-163.

2. S Iijima (1991) Helical microtubules of graphitic carbon. Nature 354: 56-58.

3. S Iijima, T Ichihashi (1993) Single-shell carbon nanotubes of 1-nm diameter. Nature 363: 603-605.

4. DS Bethune, CH Kiang, MS De Vries, G Gorman, R Savoy, et al. (1993) Cobalt-catalysed growth of carbon nanotubes with single-atomic-layer walls. Nature 363: 605-607.
5. Valentin N, Popov (2004) Carbon nanotubes: properties and application, Materials Science and Engineering R 43: 61-102.

6. Li Z, Gao B, Chen GZ, Mokaya R, Sotiropoulos S, et al. (2011) Carbon nanotube/titanium dioxide $\left(\mathrm{CNT} / \mathrm{TiO}_{2}\right)$ core-shell nanocomposites with tailored shell thickness, CNT content and photocatalytic/ photoelectrocatalytic properties. Appli Catalysis B: Environmental 110(2): 50-57.

7. Shakiba Sadeghian, Mohammad Reza Khanlari (2014) Synthesis and Characterization the Photocatalytic Activity of $\mathrm{CNT} / \mathrm{TiO}_{2}$ NanoComposite, International Journal of Applied Physics and Mathematics 4(5): 363-370.

8. Hu JS, Ren LL, Guo YG, Han-Pu Liang, An-Min Cao, et al. (2005) Mass production and high photocatalytic activity of $\mathrm{ZnS}$ nanoporous nanoparticles. Angew Chem Int Edition 44(1): 1269-1273.

9. Kalpna Varshney (2014) Carbon Nanotubes: A Review on Synthesis, Properties and Applications. International Journal of Engineering Research and General Science 2(4): 660-677.

10. H Dai, A Javey, E Pop, D Mann, Y Lu, et al. (2006) Electrical transport properties and field-effect transistors of carbon nanotubes. NANO: Brief Reports and Reviews 1(1): 1-4.

11. E Pop, D Mann, Q Wang, K Goodson, H Dai (2006) Thermal conductance of an individual single-wall carbon nanotube above room temperature. Nano Letters 6(1): 96-100.

12. Stahl H, J Appenzeller, R Martel, P Avouris, B Lengeler (2000) Intertube coupling in ropes of single-wall carbon nanotubes. Physical Review Letters 85(24): 5186-5189.

13. Hoffmann MR, Martin ST, Choi WY, Bahnemann DW (1995) Environmental Applications of Semiconductor Photocatalysis. Chemical Reviews 95(1): 69-96.

14. Kongkanand A, Kamat PV (2007) Electron storage in single wall carbon nanotubes. Fermi level equilibration in semiconductor-SWCNT suspensions. ACS Nano 1(1): 13-21.

15. Yao Y, Li G, Ciston S, Lueptow RM, Gray KA (2008) Photoreactive $\mathrm{TiO}_{2} /$ carbon nanotube composites: Synthesis and reactivity. Environmental Science and Technology 42(13): 4952-4957.

16. Yu Y, Yu JC, Yu JG, Kwok YC, YK Zhao, et al. (2005) Enhancement of photocatalytic activity of mesoporous $\mathrm{TiO}_{2}$ by using carbon nanotubes. Applied Catalysis A: General 289(2): 186-196.

17. Yu Y, Yu JC, Chan CY, YK Zhao, JC Ding, et al. (2005) Enhancement of adsorption and photocatalytic activity of $\mathrm{TiO}_{2}$ by using carbon nanotubes for the treatment of azo dye. Applied Catalysis B: Environmental 61(12):1-11.

18. Silva CG, Faria JL (2010) Photocatalytic oxidation of benzene derivatives in aqueous suspensions: Synergic effect induced by the introduction of carbon nanotubes in a $\mathrm{TiO}_{2}$ matrix. Applied Catalysis, B: Environmental 101(1-2): 81-89.

19. Martinez C, Canle M, Fernandez MI, Santaballa JA, Faria J (2011) Kinetics and mechanism of aqueous degradation of carbamazepine by heterogeneous photocatalysis using nanocrystalline $\mathrm{TiO}_{2}, \mathrm{ZnO}$ and multi-walled carbon nanotubes-anatase composites. Applied Catalysis, B: Environmental 102(3-4): 563-571.

20. Wang H, Wang HL, Jiang WF, Li ZQ (2009) Photocatalytic degradation of 2,4-dinitrophenol (DNP) by multi-walled carbon nanotubes (MWCNTs)/ $\mathrm{TiO}_{2}$ composite in aqueous solution under solar irradiation. Water Research 43(1): 204-210.

21. Lu SY, Tang CW, Lin YH, Kuo HF, Lai YC, et al. (2010) TiO -coated carbon nanotubes: A redshift enhanced photocatalysis at visible light. Applied Physics Letters 96(23): 231915.

22. Wang WD, Serp P, Kalck P, Faria JL (2005) Visible light photodegradation of phenol on $\mathrm{MWNT}^{-} \mathrm{TiO}_{2}$ composite catalysts prepared by a modified 
sol-gel method. Journal of Molecular Catalysis a-Chemical 235(1-2): 194-199.

23. Shakiba Sadeghian, Mohammad Reza Khanlari (2014) Synthesis and Characterization the Photocatalytic Activity of CNT/TiO NanoComposite, International Journal of Applied Physics and Mathematics 4(5): 363-370.

24. Eder D, Windle AH (2008) Carbon-inorganic hybrid materials: The carbonnanotube/ $\mathrm{TiO}_{2}$ interface. Advanced Materials 20(9): 1787-1793.

25. Ma LL, Sun HZ, Zhang YG, Lin YL, Li JL, et al. (2008) Preparation characterization and photocatalytic properties of CdS nanoparticles dotted on the surface of carbon nanotubes. Nanotechnology 19(11):115709.

26. Zhang LL, Xiong ZG, Zhao XS (2010) Pillaring Chemically Exfoliated Graphene Oxide with Carbon Nanotubes for Photocatalytic Degradation of Dyes under Visible Light Irradiation. ACS Nano 4(11): 7030-7036.

27. Li SW, Yu XL, Zhang GJ, Ma Y, Yao JN, et al. (2011) Green chemical decoration of multiwalled carbon nanotubes with polyoxometalate- encapsulated gold nanoparticles: visible light photocatalytic activities. Journal of Materials Chemistry 21(7): 2282-2287.

28. Yu Y, Yu JC, Yu JG, Kwok YC, YK Zhao, et al. (2005) Enhancement of photocatalytic activity of mesoporous $\mathrm{TiO}_{2}$ by using carbon nanotubes. Applied Catalysis A: General 289(2): 186-196.

29. Yan Y, Sun HP, Yao PP, Kang SZ, Mu J (2011) Effect of multi-walled carbon nanotubes loaded with Ag nanoparticles on the photocatalytic degradation of rhodamine B under visible light irradiation. Applied Surface Science 257(8): 3620-3626.

30. Shou-ai F, Zhen-ping ZHU, Jiang-hong ZHAO (2008) The manufacture of carbon nanotubes decorated with ZnS to enhance the ZnS photocatalytic activity. New Carbon Materials 23(3): 228-234.

31. Serp P, Corrias M, Kalck P (2003) Carbon nanotubes and nanofibers in catalysis. Appl Catal A-Gen 253(2): 337-358.

32. Wei Fei, Zhang Qiang, Qian Wei-zhong, et al. (2007) Progress on aligned carbon nanotube arrays. J. New Carbon Materials 22(3): 271-282.
(C) Commons Attribution 4.0 License

To Submit Your Article Click Here:

Submit Article
DOI: 10.32474/ANOAJ.2018.01.000115

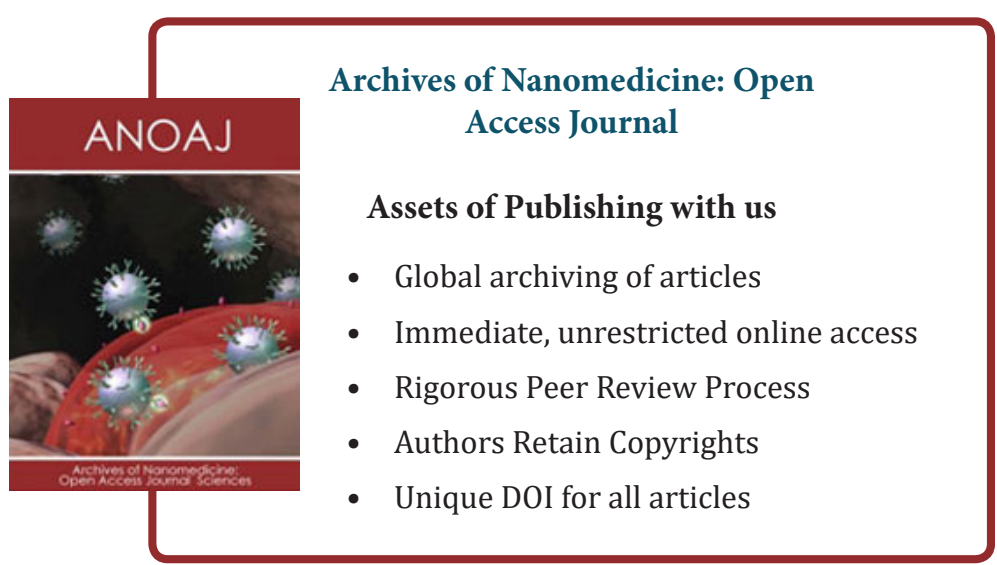

